\title{
Elevated expression of PTCD3 correlates with tumor progression and predicts poor prognosis in patients with prostate cancer
}

\author{
YIQIAO HUANG ${ }^{1}$, GANGGANG JIANG ${ }^{1}$, XUE LIANG $^{2}$, ZIQUAN LAN ${ }^{1}$, ZHENGMING SU $^{1}$, \\ HUALING WU ${ }^{1}$, JINSHENG WENG ${ }^{2}$ and XIANHAN JIANG ${ }^{1}$
}

\begin{abstract}
Departments of ${ }^{1}$ Urology and ${ }^{2}$ Central Laboratory, The Fifth Affiliated Hospital of Guangzhou Medical University, Guangzhou, Guangdong 510700, P.R. China
\end{abstract}

Received May 3, 2018; Accepted August 6, 2018

DOI: $10.3892 / \mathrm{mmr} .2018 .9402$

\begin{abstract}
Pentatricopeptide repeat domain protein 3 (PTCD3) is a mitochondrial RNA-binding protein that serves a role in mitochondrial translation. PTCD3 was originally reported as an oncogene that is involved in breast cancer and lymphoma. However, the expression and function of PTCD3 in prostate cancer $(\mathrm{PCa})$ are unknown. Therefore, the aim of the present study was to investigate the expression of PTCD3 and its clinical significance in PCa. Immunohistochemistry and dataset analyses revealed that PTCD3 protein expression levels were enhanced in human PCa tissues and mouse PCa models. PTCD3 expression levels were positively correlated with advanced $\mathrm{PCa}$ pathological grade and clinical stage. Additionally, PTCD3 mRNA expression was positively correlated with tissue malignancy, high Gleason score and distant metastasis in The Cancer Genome Atlas dataset. Kaplan-Meier analysis revealed that high PTCD3 levels can predict the increased biochemical recurrence (BCR)-free survival in all patients with or without metastasis. The overexpression of PTCD3 could be used as an independent prognostic marker of poor BCR-free survival. Immunofluorescence and western blot analysis in human $\mathrm{PCa}$ cell lines further confirmed that PTCD3 levels were associated with the hormone independence of PCa. Therefore, the present study revealed that PTCD3 levels may serve as a novel biomarker for PCa prognosis.
\end{abstract}

Correspondence to: Dr Xianhan Jiang, Department of Urology, The Fifth Affiliated Hospital of Guangzhou Medical University, 621 Gangwan Road, Guangzhou, Guangdong 510700, P.R. China E-mail: jiangxianhan@gzhmu.edu.cn

Professor Jinsheng Weng, Department of Central Laboratory, The Fifth Affiliated Hospital of Guangzhou Medical University, 621 Gangwan Road, Guangzhou, Guangdong 510700, P.R. China E-mail: jweng35@yahoo.com

Key words: pentatricopeptide repeat domain protein 3, biochemical recurrence-free survival, prognosis predictor, tumor progression, prostate cancer

\section{Introduction}

Prostate cancer (PCa) is the most commonly diagnosed male cancer in Western countries and the second leading cause of death in the US (1). Approximately 164,690 people are diagnosed with $\mathrm{PCa}$, which cause approximately $9 \%$ of cancer-related deaths among American men $(1,2)$. PCa is a heterogeneous and multi-focal cancer with highly variable natural history, causing difficulty in predicting its initiation, progression and prognosis. The combination of Gleason score, TNM stage, lymph node status and serum prostate-specific antigen (PSA) level has been commonly used to predict the prognosis of patients with PCa $(3,4)$. PSA testing is widely used for the early clinical diagnosis of PCa, but it's true value remains controversial because of its poor tumour specificity (3). According to statistics, PSA testing may have led to false positives in $23-42 \%$ of all diagnosed cases; furthermore, the patients receiving treatments, such as radical prostatectomy or radiotherapy, must pay substantial costs and subject themselves to serious side effects $(5,6)$. Approximately $25 \%$ of patients with $\mathrm{PCa}$ suffer from biochemical recurrence (BCR) following radical prostatectomy (7). Therefore, identifying methods that can be used to correctly distinguish indolent and aggressive cancers and finding novel and effective biomarkers are critical to improve the understanding of the biological progression of $\mathrm{PCa}$ and to advance the diagnosis and prognosis of $\mathrm{PCa}$ in clinics.

Mitochondrial dysfunction is closely related with cancer development (8-10). Cancer cells usually have hyperexpression of mitochondrial genes, such as the leucine-rich pentatricopeptide repeat containing (LRPPRC) gene that we have reported previously $(11,12)$. Mitochondrial genes play direct role in regulating cancer proliferation, survival and therapeutic effect $(13,14)$.

Pentatricopeptide repeat domain protein 3 (PTCD3), a mitochondrial ribosomal protein, is located on the human chromosome 2p11.2-p12 and encodes a polypeptide of 689 amino acids with an estimated molecular weight of $79 \mathrm{kDa}(15)$. PTCD3 is linked with mt-rRNA from the small ribosomal subunit and plays a role in RNA binding and mitochondrial gene expression. Some of its related pathways are mitochondrial translation and organelle biogenesis and maintenance (16). PTCD3 knockdown decreases mitochondrial respiration and 
the activity of respiratory complexes (17). Depleting PTCD3 in 143B osteosarcoma cells generally affects mitochondrial protein synthesis (16). Its prognostic value in breast cancer and its ability to act as a therapeutic target in lymphomas have been found in recent work $(18,19)$. However, few studies have been conducted to clarify the function of PTCD3 in human cancer, and the role of PTCD3 in PCa remains unknown. Therefore, we aimed to investigate PTCD3 expression in PCa tissues and examine its clinical significance in this specific malignancy.

\section{Materials and methods}

Patient tissue samples. For IHC analysis, a tissue microarray (TMA, n=78) with detailed clinical information including $71 \mathrm{PCa}$ tissues, 3 adjacent normal prostate tissue and 4 normal prostate tissue was purchased from Xi'an Alenabio, Co., Ltd., (cat no: PR803c). All patients whose prostate tissue samples were included in the TMA had not received chemotherapy or radiotherapy before the surgery and have not been diagnosed with any additional malignancies. Meanwhile, we got TCGA dataset information at https://portal.gdc.cancer.gov/, and used Perl programming language to merge the individual sample expression files. Then we transformed the ENSemble ID into symbol ID, and finally got the clinical data and mRNA sequence. The clinical information of the TCGA public dataset including $498 \mathrm{PCa}$ tissues and 52 normal prostate tissues was used to investigate the expression of PTCD3 at mRNA level and for survival analysis. Detailed information on the clinical parameters of all patients in this study is presented in Table I.

Mouse prostate tissue samples. PCa tissues from 11 month-old Prostate-specific PTEN knockout $\left(\mathrm{PTEN}^{-/}\right)$and 7 month-old TRAMP mice were collected as reported previously $(11,12)$ and gifted to the present study by Dr. Fen Wang (Institute of Biosciences and Technology, Texas A\&M Health Science Center, Houston, TX, USA). Normal prostate tissues were collected from wild-type mice aged 5 or 11 months. PCa tissues from TRAMP model mice which showed metastasis to the liver were collected at the same age of 13 months.

Immunohistochemistry (IHC) analysis in tissue microarray. The tissue microarray was deparaffinized with xylene and rehydrated for further H\&E or peroxidase (DAB) immunohistochemistry staining employing a DAKO EnVision System (Agilent Technologies, Inc., Santa Clara, CA, USA). Following a brief proteolytic digestion and a peroxidase blocking of tissue slides, the slides were incubated overnight with the primary antibodies against PTCD3 (cat no: bs-4424R, at a dilution of $1: 300$ ) at $4^{\circ} \mathrm{C}$. After washing, slides were incubated with second antibody labeled by HRP (rabbit). Finally, visualization was performed by 3,30-diaminoben-zidine tetrahydrochloride (DAB) and counterstained by hematoxylin. In each immunohistochemistry run, negative controls were stained with isotype-matched control IgG. The final immunoreactivity scores (IRS) of each case were calculated by multiplying the two scores for the immunostaining intensity and immunostaining percentage.

Immunohistochemistry staining in mouse prostate tissue samples. Normal prostate tissues collected from wild-type mice and PCa tissues collected from $\mathrm{PTEN}^{-/}$mice or TRAMP model mice were subjected to immunohistochemistry staining, and the histological scores were assigned by 2 independent clinical pathologists in a double-blinded manner. Prostate tissue samples from TRAMP, $\mathrm{PTEN}^{-/}$and wild-type mice were prepared for immunohistochemistry staining as described for LRPPRC analysis previously (12). Antibody against PTCD3 (cat no: bs-4424R) was purchased from Beijing Biosynthesis Biotechnology, Co., Ltd., (Beijing, China).

Cell culture. Two human PCa cell lines (DU145 and LNCaP) and one benign prostate cell line (RWPE-1) were purchased from American Type Culture Collection (Manassas, VA, USA). DU145 and LNCaP cell lines were maintained in the 1640 Medium (HyClone; GE Healthcare Life Sciences, Logan, UT, USA) supplemented with $10 \%$ fetal bovine serum, and RWPE-1 cells were cultured in DMEM Medium (HyClone; GE Healthcare Life Sciences) supplemented with $10 \%$ fetal bovine serum. The cells were cultured at $37^{\circ} \mathrm{C}$ in a humidified incubator with $5 \% \mathrm{CO}_{2}$.

Western blot analysis. Cells were collected and lysed in RIPA buffer containing broad protease inhibitor cocktail (EASY packs; Roche Diagnostics, Basel, Switzerland). The total protein concentration in the supernatants collected after centrifugation was measured by bicinchoninic acid (BCA). Equal amount of total protein $(40 \mu \mathrm{g})$ was loaded on and separated by SDS-PAGE (Bio-Rad Laboratories, Inc., Hercules, CA, USA), and blotted with primary antibodies and corresponding horse-radish peroxidase-conjugated secondary antibodies. GAPDH was used as an internal loading control. The primary antibodies anti-PTCD3 (cat. no: ab52099, dilution 1:1,000; Hangzhou MultiSciences (Lianke) Biotech, Co., Ltd., Zhejiang, China) and anti-GAPDH (cat. no: 5174, dilution 1:2,000; Cell Signaling Technology, Inc., Danvers, MA, USA) were used. Protein bands were visualized with the GelDoc XR + chemiluminescent detection system (Bio-Rad Laboratories, Inc.). Densitometric analysis of the bands was performed using the ImageJ free software (National Institutes of Health, Bethesda, MD, USA).

Immunofluorescence. Cells were digested and grown on glass coverslips for $6 \mathrm{~h}$. Then the cells were washed three times with PBS, fixed in 4\% PFA for $20 \mathrm{~min}$ and permeabilized with $0.1 \%$ Triton X-100 in PBS for 20 min. The cells were washed three times with PBS and blocked with $2 \%$ BSA in PBS for 30 min to $1 \mathrm{~h}$ at room temperature. Cells were incubated overnight at $4^{\circ} \mathrm{C}$ with anti-PTCD3 (cat. no: bs-4424R; dilution 1:200) in $1 \%$ BSA in PBS followed by five-times washing with PBS. Cells were incubated with DYLight649 goat anti-rabbit IgG [H+L] secondary antibody (GAR6492; Hangzhou MultiSciences (Lianke) Biotech, Co., Ltd.) at a 1:500 dilution in PBS at room temperature for $1 \mathrm{~h}$. After washing with PBS for five times, cells were incubated with DAPI for $5 \mathrm{~min}$ followed by five-times washing with PBS. Cells were then mounted with Fluoromount-G (Invitrogen; Thermo Fisher Scientific, Inc., Waltham, MA, USA), and images were captured using a confocal laser scanning microscope (LSM800) equipped with a $x 40$ objective. 
Table I. Expression of PTCD3 and its association with clinicopathologic features in prostate cancer.

\begin{tabular}{|c|c|c|c|c|c|c|c|}
\hline \multirow[b]{2}{*}{ Clinical features } & \multirow[b]{2}{*}{ Case } & \multicolumn{3}{|c|}{ TMA } & \multicolumn{3}{|c|}{ TCGA } \\
\hline & & Low, n (\%) & High, n (\%) & P-value & Case & $\overline{\mathrm{X}} \pm \mathrm{S}$ & P-value \\
\hline \multicolumn{8}{|l|}{ Tissue } \\
\hline Cancer & 71 & $31(43.7)$ & $40(56.3)$ & \multirow[t]{2}{*}{1.000} & 498 & $911.22 \pm 222.35$ & \multirow[t]{2}{*}{$0.011^{\mathrm{a}}$} \\
\hline Benign & 7 & $3(42.9)$ & $4(57.1)$ & & 52 & $859.94 \pm 122.77$ & \\
\hline \multicolumn{8}{|l|}{ Age, years } \\
\hline$<60$ & 5 & $4(80.0)$ & $1(20.0)$ & \multirow[t]{2}{*}{0.160} & 201 & $897.94 \pm 198.71$ & \multirow[t]{2}{*}{0.266} \\
\hline$\geq 60$ & 66 & $27(40.9)$ & $39(59.1)$ & & 296 & $920.60 \pm 237.22$ & \\
\hline \multicolumn{8}{|l|}{ Gleason score } \\
\hline$<7$ & - & - & - & \multirow[t]{3}{*}{-} & 44 & $866.50 \pm 105.94$ & \multirow[t]{3}{*}{$<0.001^{\mathrm{b}}$} \\
\hline$=7$ & & & & & 247 & $876.59 \pm 178.93$ & \\
\hline$>7$ & - & - & - & & 206 & $962.83 \pm 272.90$ & \\
\hline \multicolumn{8}{|c|}{ Serum PSA levels (ng/ml) } \\
\hline$<10$ & - & - & - & \multirow[t]{2}{*}{-} & 8 & $1041.54 \pm 287.19$ & \multirow[t]{2}{*}{0.109} \\
\hline$\geq 10$ & - & - & - & & 438 & $912.02 \pm 225.04$ & \\
\hline \multicolumn{8}{|l|}{ Pathological grade } \\
\hline$\leq 2$ & 23 & $16(69.6)$ & $7(30.4)$ & \multirow[t]{2}{*}{$0.001^{\mathrm{b}}$} & - & - & \multirow[t]{2}{*}{-} \\
\hline$>2$ & 44 & $12(27.3)$ & $32(72.7)$ & & - & - & \\
\hline \multicolumn{8}{|l|}{ Clinical stage } \\
\hline I & 4 & $4(100.0)$ & $0(0.0)$ & \multirow[t]{2}{*}{$0.034^{\mathrm{a}}$} & - & - & \multirow[t]{2}{*}{-} \\
\hline II-IV & 66 & 27 (40.9) & $39(59.1)$ & & - & - & \\
\hline \multicolumn{8}{|l|}{ Tumor invasion } \\
\hline $\mathrm{T} 1-\mathrm{T} 2$ & 46 & $22(47.8)$ & $24(52.2)$ & \multirow[t]{2}{*}{0.409} & 351 & $887.35 \pm 188.31$ & \multirow[t]{2}{*}{0.076} \\
\hline $\mathrm{T} 3-\mathrm{T} 4$ & 24 & $9(37.5)$ & $15(62.5)$ & & 55 & $952.75 \pm 258.09$ & \\
\hline \multicolumn{8}{|c|}{ Lymph node metastasis } \\
\hline No & 58 & $25(43.1)$ & $33(56.9)$ & \multirow[t]{2}{*}{0.662} & 344 & $909.60 \pm 212.73$ & \multirow[t]{2}{*}{0.384} \\
\hline N1 & 12 & $6(50.0)$ & $6(50.0)$ & & 80 & $933.45 \pm 251.57$ & \\
\hline \multicolumn{8}{|l|}{ Distant metastasis } \\
\hline M0 & 56 & $24(42.9)$ & $32(57.1)$ & 0.630 & 455 & $907.32 \pm 222.61$ & $0.002^{\mathrm{b}}$ \\
\hline M1 & 14 & $7(50.0)$ & $7(50.0)$ & & 3 & $1307.89 \pm 492.13$ & \\
\hline Survival time, ye & & & & & & & \\
\hline$<5$ & - & - & - & - & 411 & $911.86 \pm 196.51$ & 0.946 \\
\hline$\geq 5$ & - & - & - & & 86 & $909.44 \pm 320.39$ & \\
\hline
\end{tabular}

${ }^{\mathrm{a}} \mathrm{P}<0.05$ and ${ }^{\mathrm{b}} \mathrm{P}<0.01$. Product $>4$ : High; Product $\leq 4$ : Low. $\mathrm{PTCD} 3$, pentatricopeptide repeat domain protein 3 ; '-', no information for patients in the cohort; PSA, prostate-specific antigen; TMA, tissue microarray; TCGA, The Cancer Genome Atlas.

Statistical analysis. SPSS v.22.0 software (IBM Corp., Armonk, NY, USA) was used for statistical analysis. Pearson's Chi-squared tests and Fisher's exact test were used to analyze the association of PTCD3 protein expression with clinicopathological parameters. The association between PTCD3 mRNA expression with patient's clinicopathological was analyzed by Student's t-test (pairwise comparison) and one-way analysis of variance plus least significant difference post-hoc test (inter-group comparison). Kaplan-Meier curve method was used for survival analysis, and the differences were assessed by log-rank test. Further Univariate analysis comparisons and multivariate survival analysis comparisons were obtained by using Cox proportional hazards regression.
The relative risks of mortality were expressed as adjusted hazard ratios (HRs) and their corresponding 95\% confidence intervals (CIs). $\mathrm{P}<0.05$ was considered to indicate a statistically significant difference.

\section{Results}

The expression of PTCD3 protein is increased in PCa and is associated with aggressive phenotypes. We first detected the expression of PTCD3 protein in 71 PCa tissues, 3 adjacent normal prostate tissues and 4 normal prostate tissues by IHC analysis (Table I). As shown in Fig. 1A-D, PTCD3 immunostainings appeared strongly in PCa cancer cells in PCa 


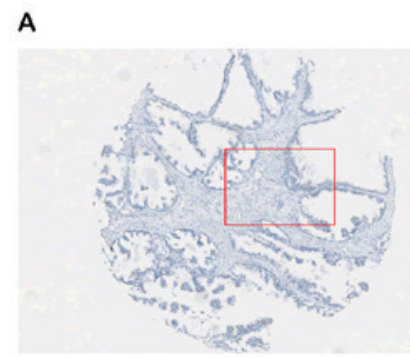

Normal

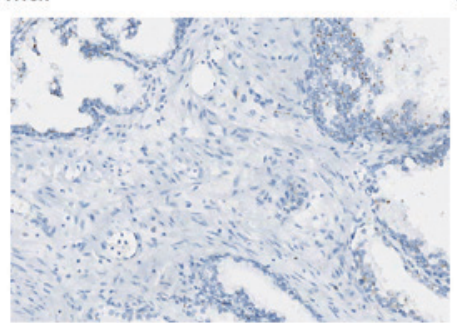

Cancer

C

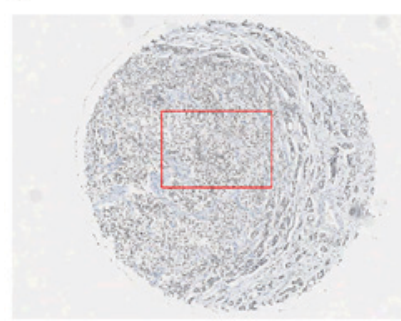

B

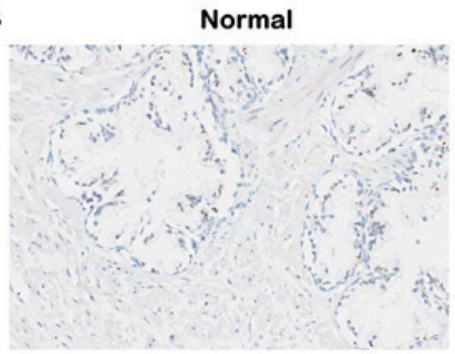

D

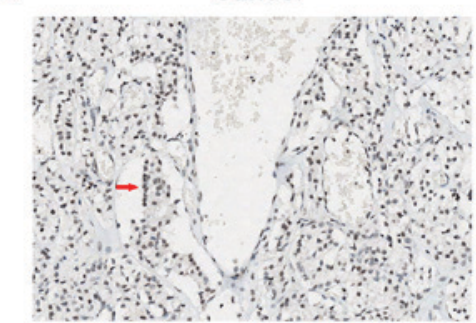

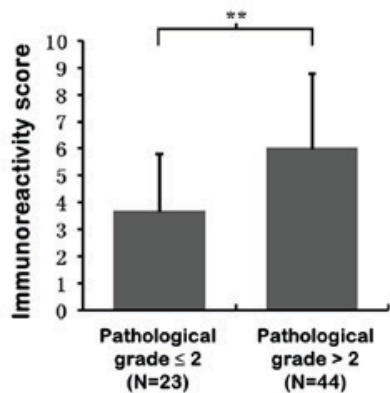

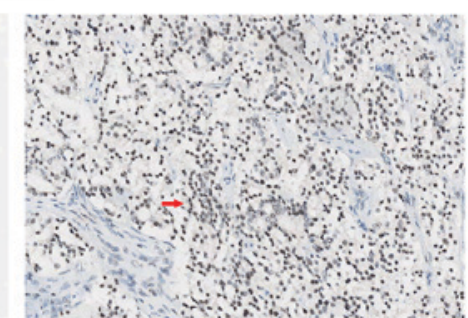

F

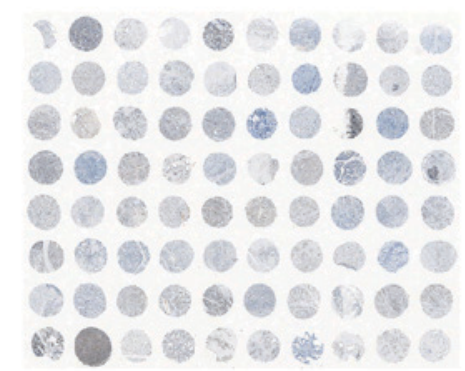

Figure 1. Immunohistochemical staining for PTCD3 in PCa and normal prostate tissues (magnification, x100 and x400). (A and B) Representative images presenting the (A) negative or (B) weak immunostainings of PTCD3 protein in non-cancerous prostate tissues. (C and D) Representative images presenting the strong immunostainings of PTCD3 protein in PCa tissues. (E) A plot of the immunoreactivity score of PTCD3 protein in PCa tissues indicating the difference between pathological grade $\leq 2$ and pathological grade $>2$. (F) The scanned images of the whole TMA. Arrows indicate positively stained cells. ${ }^{* *} \mathrm{P}<0.01$, as indicated. PTCD3, pentatricopeptide repeat domain protein 3; PCa, prostate cancer.

tissues but weakly in normal prostate tissues. Overexpression of PTCD3 protein was significantly related to advanced $\mathrm{PCa}$ pathological grade (IRS $3.70 \pm 2.10$ for pathological grade $\leq 2$ vs. $6.03 \pm 2.74$ for pathological grade $>2$; Fig. 1E) and higher clinical stages of PCa tissues (Table I). However, the levels of PTCD3 were not associated with age, distant metastasis, lymph node metastasis and tumour invasion (Table I).

PTCD3 is overexpressed in PCa and is correlated with gleason score and distant metastasis of PCa in TCGA dataset. Publicly available data from the TCGA dataset, which consists of $498 \mathrm{PCa}$ tissues and 52 normal prostate tissue with high-throughput sequencing data, were used to validate the expression data of protein-coding genes (mRNA) (20). As shown in Table I, PTCD3 was overexpressed in the PCa tissue samples $(\mathrm{P}=0.011)$ compared with that in benign prostate tissues samples. Patients with $\mathrm{PCa}$ exhibiting high Gleason score $(\mathrm{P}<0.001)$ and increased distant metastasis $(\mathrm{P}=0.002)$ showed high PTCD3 mRNA expression levels. However, high PTCD3 expression was not associated with age, serum PSA levels, tumour invasion, lymph node metastasis, and survival time in the TCGA dataset $(\mathrm{P}>0.05)$.

PTCD3 expression is elevated in the tissue samples of prostate-specific PTEN $N^{--}$mouse model and TRAMP model.
To confirm the elevated PTCD3 expression in PCa tissues and investigate the relationship between PTCD3 and tumour progression, we further performed immunohistochemical (IHC) analysis in the tissue samples of the prostate-specific $\mathrm{PTEN}^{-/-}$mouse model and TRAMP model.

Similar to the results in human PCa tissues, PTCD3 levels were low in normal prostate tissues of WT mice but were elevated in PCa samples from the PTEN knockout mice and TRAMP model (Fig. 2). The PTCD3 levels between the normal prostate tissues of WT mice and PCA in PTEN-deficient mice or TRAMP model were significantly different $(\mathrm{P}<0.001$; Fig. 2J). We found that PTCD3 expression was significantly higher in PCa tissue from the 13-month-old TRAMP mouse model, which exhibited metastasis to the liver, than in the other mouse samples. These data from mouse PCa tissues were consistent with previous results in human $\mathrm{PCa}$ and further confirmed that the levels of PTCD3 were correlated with the aggressive progression of $\mathrm{PCa}$.

Overexpression of PTCD3 is correlated with Hormone-independence of human PCa. Given that the prostate is a hormone-regulated organ, early-stage $\mathrm{PCa}$ is usually hormone dependent, whereas late-stage PCa only becomes hormone independent after androgen depletion treatment. We extracted proteins from two human PCa cell lines (DU145 and LNCaP) 


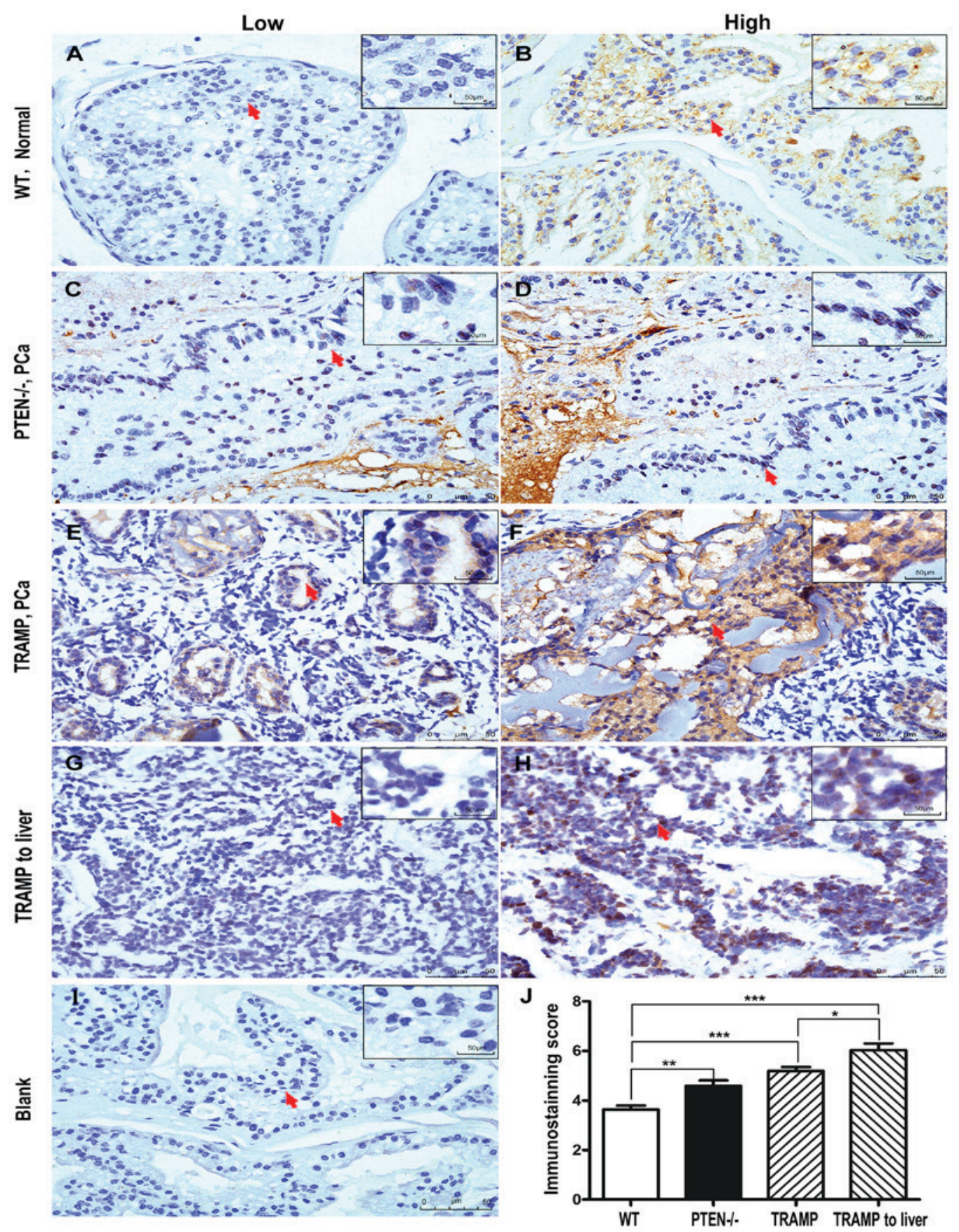

Figure 2. Immunohistochemical staining for PTCD3 in normal mouse prostate tissues and prostate tumors of different mouse PCa models. (A and B) Representative images of normal prostate tissue samples of wide-type mice presenting (A) low or (B) high levels of PTCD3. (C and D) Representative images of PCa tissue samples of PTEN-/- mice showing (C) low or (D) high levels of PTCD3. (E and F) Representative images of PCa tissue samples of TRAMP model presenting (E) low or (F) high levels of PTCD3. (G and H) Representative images of PCa tissues collected from TRAMP mice with metastasis to the liver presenting $(\mathrm{G})$ low or $(\mathrm{H})$ high levels of PTCD3. (I) Representative images of PCa tissues without staining of PTCD3 as a negative control. Scale bars, $50 \mu \mathrm{m}$; magnification, $\mathrm{x} 400$ and $\mathrm{x} 800$. (J) A plot of the immunoreactivity score of PTCD3 protein in different mouse prostate tissues. Arrows indicate positively stained cells. ${ }^{*} \mathrm{P}<0.05,{ }^{* *} \mathrm{P}<0.01$ and ${ }^{* * *} \mathrm{P}<0.001$, as indicated. PTCD3, pentatricopeptide repeat domain protein 3 ; PCa, prostate cancer; PTEN, phosphatase and tensin homolog; TRAMP, transgenic adenocarcinoma of the mouse prostate.

and one benign prostate cell line (RWPE-1) to determine the levels of PTCD3 in different stages of PCa. We found that the levels of PTCD3 were lower in normal prostate cells than in PCa cells, and the expression levels of PTCD3 in hormone-dependent $\mathrm{PCa}$ cells $(\mathrm{LnCaP})$ were lower than in hormone-independent PCa cells (DU145) (Fig. 3A and B). This finding indicates that the levels of PTCD3 were higher in hormone-independent PCa, which is usually detected in patients with late-stage disease. To confirm this result, we further performed immunofluorescence assays in these three cell lines. We found that the expression levels of PTCD3 were not detectable in RWPE-1 cell but were significantly high in PCa cells including LNCaP and DU145 cells (Fig. 3C and D). Similar to the results of Western blot analysis, the fluorescence intensities of PTCD3 in DU145 cells were brighter than those in LNCaP and RWPE-1 cells (Fig. 3C and D). The data from normal prostate cells and PCa cells further confirmed that the levels of PTCD3 were correlated with the hormone independence of human PCa. 
A

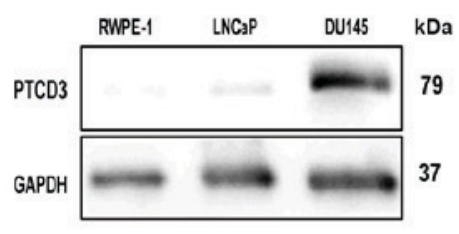

B
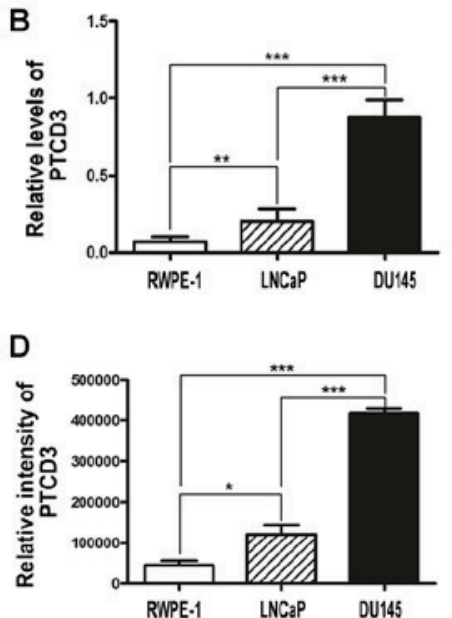

C
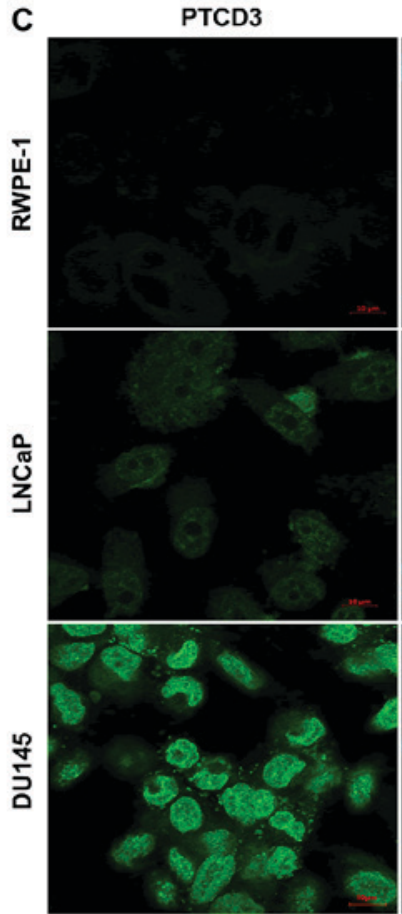
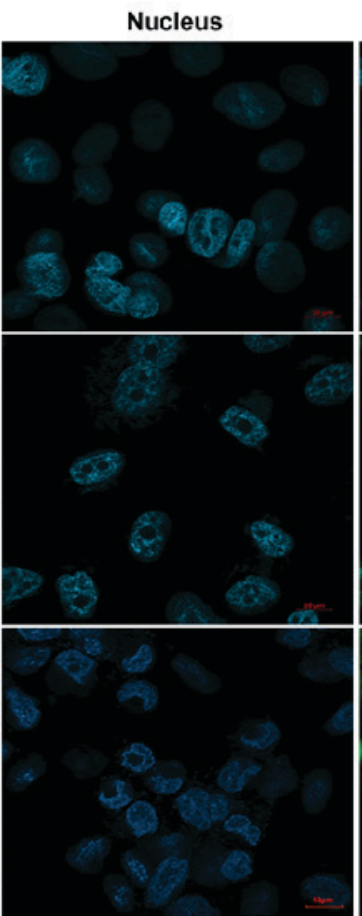

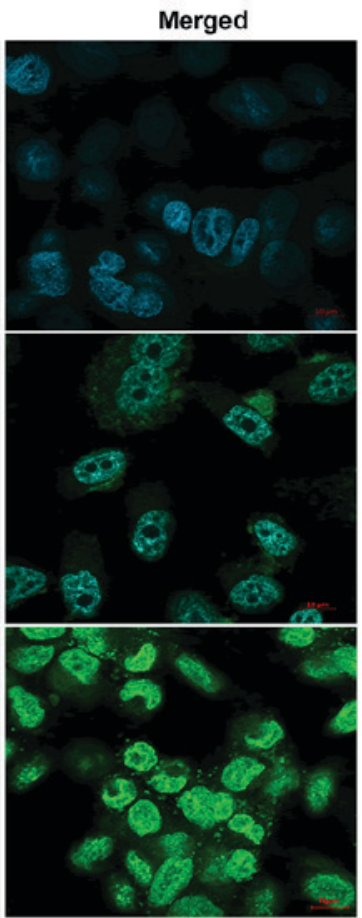

Figure 3. Expression of PTCD3 protein in cultured normal prostate cells (RWPE-1) and human PCa cell lines (LNCaP and DU145). (A) Western blot analysis of the expression levels of PTCD3 in the RWPE-1, LNCaP and DU145 cell lines. (B) A plot of relative immunoblot levels of PTCD3 in RWPE-1, LNCaP and DU145 cell lines. (C) Immunofluorescence assays of PTCD3 expression in RWPE-1, LNCaP and DU145 cell lines. Scale bars, $10 \mu$ m. (D) A plot of relative fluorescence intensity of PTCD3 in the RWPE-1, LNCaP and DU145 cell lines. ${ }^{*} \mathrm{P}<0.05,{ }^{* *} \mathrm{P}<0.01$ and ${ }^{* * *} \mathrm{P}<0.001$, as indicated. PTCD3, pentatricopeptide repeat domain protein 3 ; $\mathrm{PCa}$, prostate cancer.

Overexpression of PTCD3 is associated with poor prognosis of PCa in TCGA dataset. Overall survival and BCR-free survival are important to PCa patients. Especially, BCR-free survival is authoritative to $\mathrm{PCa}$ patients for its predictive function, which may affect the follow-up treatments. Kaplan-Meier method was used to compare the overall and BCR-free survival of patients with high and low PTCD3 expression to further evaluate the prognostic value of overexpression of PTCD3 in patients with PCa. Median PTCD3 mRNA expression in all PCa tissues was used as the cut-off point to classify all cases into PTCD3 high ( $n=210$, in TCGA dataset) and PTCD3 low ( $n=215$, in TCGA dataset) groups. Fig. 3 shows that all $\mathrm{PCa}$ patients with high PTCD3 expression had shorter overall survival (Fig. 4A) than those with low PTCD3 expression $(\mathrm{P}=0.034)$. No statistically significant difference was found between high PTCD3 and low PTCD3 groups in non-metastatic patients $(\mathrm{P}=0.058$; Fig. 4C). All PCa patients and non-metastatic patients with high PTCD3 expression showed shorter BCR-free survival $(\mathrm{P}=0.013, \mathrm{P}=0.016$; Fig. 4B and $\mathrm{D})$ than those with low PTCD3 expression.

PTCD3 can serve as an independent prognostic marker of PCa patients. We further utilized the Cox proportional hazard model to assess whether or not PTCD3 is an independent prognostic predictor for the survival of PCa patients documented in the TCGA database. In Table II, the univariate model analysis shows that the level of PTCD3 mRNA was a significant prognostic factor for BCR-free survival in patients with PCa with HR of 1.942, 95\% CI of 1.141-3.306 and $\mathrm{P}=0.014$. Multivariate analysis using Cox proportional hazard model further revealed that a high level of PTCD3 mRNA was a significant independent prognostic marker for patients with PCa (HR of $1.956,95 \%$ CI of $1.113-3.436$ and $\mathrm{P}=0.020$; Table II).

\section{Discussion}

As a global, heterogeneous, and multifocal disease, $\mathrm{PCa}$ continues to be a burden on the healthcare system (21). One in every six men can develop PCa in his lifetime, and the incidence rate increases with age (22). When PCa becomes metastatic and invasive, it manifests an extremely unfavourable prognosis that is commonly the primary cause of death. Thus, finding an effective biomarker that can distinguish between indolent and aggressive $\mathrm{PCa}$ and can predict the clinical outcome of patients with PCa may benefit patient management and decrease morbidity.

PTCD3 is one of the mammalian mitochondrial pentatricopeptide repeat (PPR) domain proteins (23) that play a critical role in mitochondrial translation and organelle biogenesis and maintenance (17,24). During carcinogenesis, the material and energy in the cells are reshaped to support and help the survival of cancer cells and to satisfy the requirements for the synthesis of biological macromolecules and energy supplement in cancer cells (25). Mitochondria are an important stress receptor in cells and the central part of all metabolic reactions (26) and thus play a critical role in tumorigenesis. Our previous studies showed that the mitochondrion-associated protein, LRPPRC, is one of the mammalian mitochondrial PPR domain proteins that prevent the degradation of the mitochondria; high LRPPRC levels can be used as an independent biomarker for patients 
Table II. Prognostic value of PTCD3 for BCR-free survival by Cox proportional hazards model.

BCR-free survival

Variables

$\operatorname{HR}(95 \% \mathrm{CI})$

P-value

Univariate analysis

Gleason score ( $<7$ vs. $=7$ vs. $>7$ )

$3.110(1.841-5.255)$

$<0.001^{\mathrm{c}}$

Serum PSA levels (<10 vs. $\geq 10)$

$9.396(3.978-22.196)$

$<0.0001^{\mathrm{d}}$

pN stage (N0 vs. N1)

$1.879(1.049-3.365)$

$0.034^{\mathrm{a}}$

PTCD3 expression (low vs. high)

$1.942(1.141-3.306)$

$0.014^{\mathrm{a}}$

Multivariate analysis

Gleason score ( $<7$ vs. $=7$ vs. $>7$ )

$2.578(1.378-4.825)$

$0.003^{\mathrm{b}}$

Serum PSA levels (<10 vs. $\geq 10$ )

$6.579(2.533-17.086)$

$<0.001^{\mathrm{c}}$

pN stage (N0 vs. N1)

$1.659(0.912-3.081)$

0.097

PTCD3 expression (low vs. high)

$1.956(1.113-3.436)$

$0.020^{\mathrm{a}}$

${ }^{\mathrm{a}} \mathrm{P}<0.05 ;{ }^{\mathrm{b}} \mathrm{P}<0.01 ;{ }^{\mathrm{c}} \mathrm{P}<0.001 ;{ }^{\mathrm{d}} \mathrm{P}<0.0001$. HR, hazard ratio; $\mathrm{CI}$, confidence interval; $\mathrm{PTCD} 3$, pentatricopeptide repeat domain protein 3 ; BCR, biochemical recurrence.

A

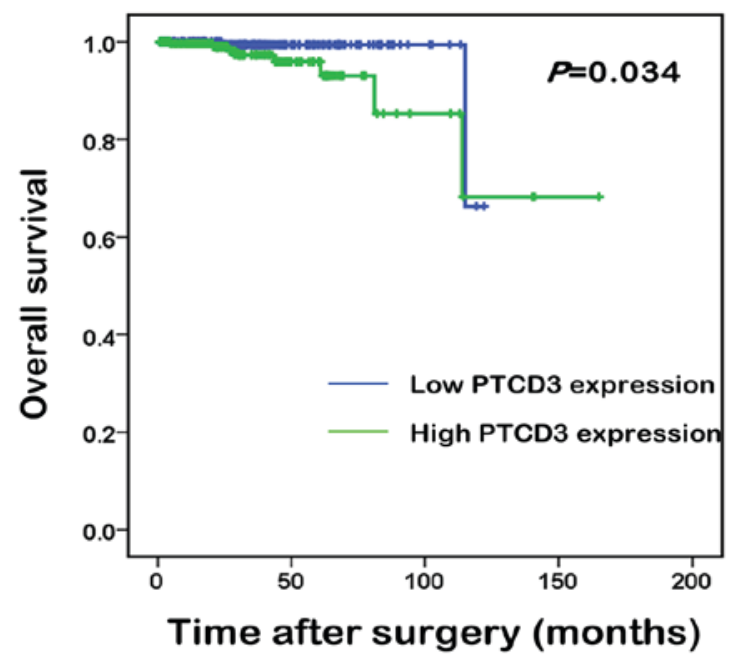

C

C Non-metastatic patients

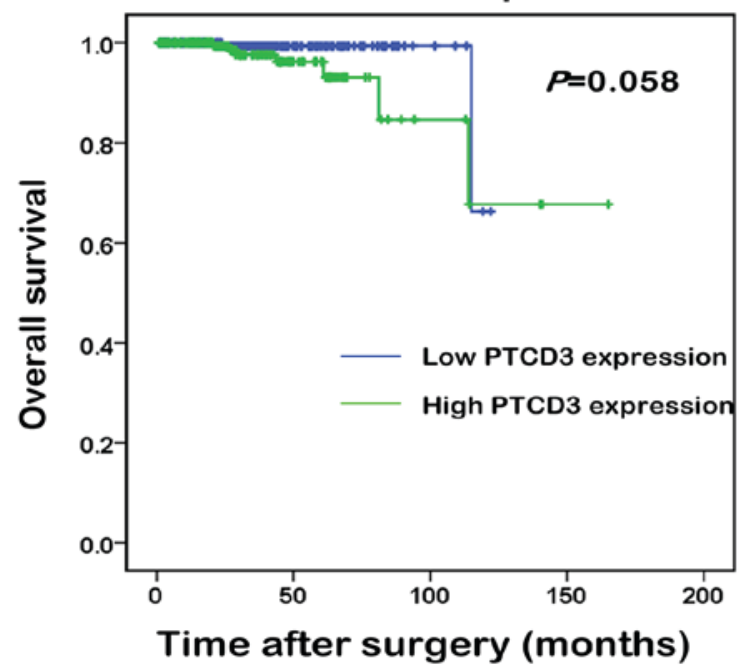

B

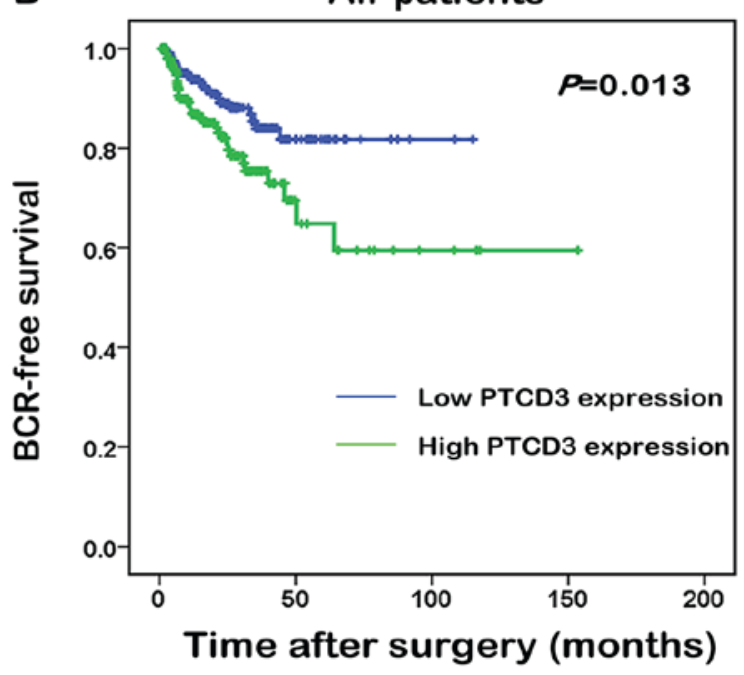

D

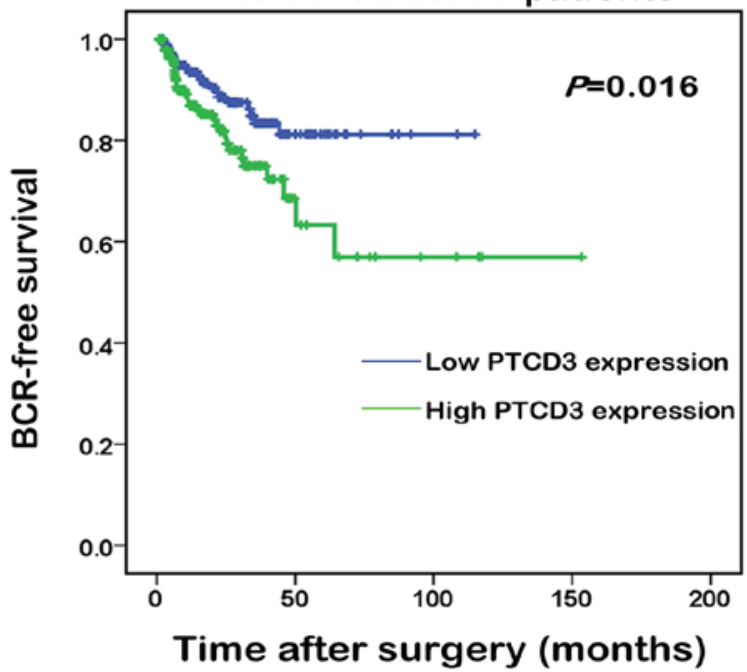

Figure 4. High levels of PTCD3 predict a poor prognosis in PCa patients. (A and B) Kaplan-Meier curves presenting the difference in (A) overall and (B) BCR-free survival between patients with high and low expression levels of PTCD3. (C and D) Kaplan-Meier curves presenting the difference in (C) overall survival and (D) BCR-free survival between non-metastatic patients with high and low expression levels of PTCD3. PTCD3, pentatricopeptide repeat domain protein 3; BCR, biochemical recurrence. PCa, prostate cancer. 
with late-stage PCa and poor prognosis $(11,12)$. Therefore, PTCD3 may have an important function in PCa progression. D'Andrea et al (19), recently identified that the inhibition of the mitochondrial translation factor PTCD3 may be detrimental to lymphomagenesis, and PTCD3 may act as a therapeutic target in lymphomas (27). However, the function of PTCD3 in human cancer was only investigated in few studies, and the clinical significance of PTCD3 in the context of human PCa has not been reported yet.

In the present study, we found that PTCD3 played an important role in PCa tumour progression by acting as an oncogene. PTCD3 expression at both mRNA and protein levels was up-regulated in the PCa tissues with advanced pathological or clinical stage. High PTCD3 expression was dramatically associated with aggressive tumour progression in patients with $\mathrm{PCa}$, including high Gleason score, short overall survival time, short BCR-free survival time and high distant metastasis. The IHC results in mouse PCa tissues also proved that elevated PTCD3 levels were positively correlated with the aggressive progression of PCa. Furthermore, the results of Western blot analysis and immunofluorescence assays performed in normal prostate cells (RWPE-1) and $\mathrm{PCa}$ cells (DU145 and LNCaP) showed that PTCD3 levels were lower in benign prostate cells than in PCa cells. By contrast, the hormone-independent DU145 PCa cells exhibited the strongest PTCD3 fluorescence intensity. These findings indicated that PTCD3 overexpression was correlated with the stages of human PCa. PTCD3 expression was identified as an unfavourable prognostic factor of BCR-free survival in patients with PCa. To our knowledge, this study is the first to investigate the role of PTCD3 in the prognosis of PCa.

In conclusion, our data strongly suggested that PTCD3 overexpression may play an important role in the characteristics and aggressive behaviour of PCa, especially in groups with advanced pathological or clinical stage and high Gleason score. Additionally, PTCD3 upregulation is associated with poor prognosis in patients with $\mathrm{PCa}$. This protein may be a novel prognostic biomarker for $\mathrm{PCa}$ and a potential therapeutic target for patients with PCa. However, further study is necessary to gain a full understanding of the underlying molecular mechanisms.

\section{Acknowledgements}

The authors would like to thank Dr. Fen Wang (Institute of Biosciences and Technology, Texas A\&M Health Science Center, Houston, TX, USA) for providing mouse prostate tissues samples.

\section{Funding}

The present study was supported by the Guangzhou Municipal Science and Technology Project (grant no. 1563000448) and National Natural Science Foundation of China (grant no. 81570189).

\section{Availability of data and materials}

The datasets used and/or analyzed during the present study are available from the corresponding author on reasonable request.

\section{Authors' contributions}

$\mathrm{XJ}, \mathrm{JW}$ and $\mathrm{YH}$ designed the study and drafted the manuscript. GJ, ZL and XL performed the experiments. YH, ZS and HW analyzed and interpreted the data. All authors have read and approved the final manuscript.

\section{Ethics approval and consent to participate}

Not applicable.

\section{Patient consent for publication}

Not applicable.

\section{Competing interests}

The authors declare that they have no competing interests.

\section{References}

1. Siegel RL, Miller KD and Jemal A: Cancer statistics, 2018. CA Cancer J Clin 68: 7-30, 2018.

2. Society AC: Cancer facts \& figures 2016. American Cancer Society, Atlanta, GA, 2016.

3. Center MM, Jemal A, Lortet-Tieulent J, Ward E, Ferlay J, Brawley $\mathrm{O}$ and Bray $\mathrm{F}$ : International variation in prostate cancer incidence and mortality rates. Eur Urol 61: 1079-1092, 2012.

4. Blute ML Jr, Damaschke NA and Jarrard DF: The epigenetics of prostate cancer diagnosis and prognosis: Update on clinical applications. Curr Opin Urol 25: 83-88, 2015.

5. Draisma G, Etzioni R, Tsodikov A, Mariotto A, Wever E, Gulati R, Feuer E and de Koning H: Lead time and overdiagnosis in prostate-specific antigen screening: Importance of methods and context. J Natl Cancer Inst 101: 374-383, 2009.

6. PSA-based screening for prostate cancer. Too many adverse effects. Prescrire Int 21: 215-217, 2012.

7. Garg AD, Martin S, Golab J and Agostinis P: Danger signalling during cancer cell death: Origins, plasticity and regulation. Cell Death Differ 21: 26-38, 2014.

8. Sehrawat A, Roy R, Pore SK, Hahm ER, Samanta SK, Singh KB, Kim SH, Singh K and Singh SV: Mitochondrial dysfunction in cancer chemoprevention by phytochemicals from dietary and medicinal plants. Semin Cancer Biol 47: 147-153, 2017.

9. Guerra F, Guaragnella N, Arbini AA, Bucci C, Giannattasio S and Moro L: Mitochondrial dysfunction: A novel potential driver of epithelial-to-mesenchymal transition in cancer. Front Oncol 7: 295, 2017.

10. Ježek J, Cooper KF and Strich R: Reactive oxygen species and mitochondrial dynamics: The yin and yang of mitochondrial dysfunction and cancer progression. Antioxidants (Basel) 7: pii: E13, 2018.

11. Jiang X, Zhong W, Huang H, He H, Jiang F, Chen Y, Yue F, Zou J, Li X, He Y, et al: Autophagy defects suggested by low levels of autophagy activator MAP1S and high levels of autophagy inhibitor LRPPRC predict poor prognosis of prostate cancer patients. Mol Carcinog 54: 1194-1204, 2015.

12. Jiang X, Li X, Huang H, Jiang F, Lin Z, He H, Chen Y, Yue F, Zou J, He Y, et al: Elevated levels of mitochondrion-associated autophagy inhibitor LRPPRC are associated with poor prognosis in patients with prostate cancer. Cancer 120: 1228-1236, 2014.

13. Li Y, Beckman KB, Caberto C, Kazma R, Lum-Jones A, Haiman CA, Le Marchand L, Stram DO, Saxena R and Cheng I: Association of genes, pathways, and haplogroups of the mitochondrial genome with the risk of colorectal cancer: The multiethnic cohort. PLoS One 10: e0136796, 2015.

14. Burch TC, Rhim JS and Nyalwidhe JO: Novel altered mitochondrial genes in prostate cancer progression. Cancer Res 75, 2015.

15. Genecards: Summaries for PTCD3 Gene. https://www.genecards. org/cgi-bin/carddisp.pl?gene=PTCD3. Accessed August 8, 2018.

16. Lightowlers RN and Chrzanowska-Lightowlers ZM: Human pentatricopeptide proteins: Only a few and what do they do? RNA Biol 10: 1433-1438, 2013. 
17. Rackham O, Davies SM, Shearwood AM, Hamilton KL, Whelan J and Filipovska A: Pentatricopeptide repeat domain protein 1 lowers the levels of mitochondrial leucine tRNAs in cells. Nucleic Acids Res 37: 5859-5867, 2009.

18. Madhavan S, Gusev Y, Singh S and Riggins RB: ERR $\gamma$ target genes are poor prognostic factors in Tamoxifen-treated breast cancer. J Exp Clin Cancer Res 34: 45, 2015.

19. D'Andrea A, Gritti I, Nicoli P, Giorgio M, Doni M, Conti A Bianchi V, Casoli L, Sabò A, Mironov A, et al: The mitochondria translation machinery as a therapeutic target in Myc-driven lymphomas. Oncotarget 7: 72415-72430, 2016.

20. Cancer Genome Atlas Research Network: Comprehensive molecular profiling of lung adenocarcinoma. Nature 511: 543-550, 2014.

21. Esfahani M, Ataei N and Panjehpour M: Biomarkers for evaluation of prostate cancer prognosis. Asian Pac J Cancer Prev 16: 2601-2611, 2015.

22. Baade PD, Youlden DR and Krnjacki LJ: International epidemiology of prostate cancer: Geographical distribution and secular trends. Mol Nutr Food Res 53: 171-184, 2009.
23. Manna S: An overview of pentatricopeptide repeat proteins and their applications. Biochimie 113: 93-99, 2015.

24. Baggio F, Bratic A, Mourier A, Kauppila TE, Tain LS, Kukat C, Habermann B, Partridge L and Larsson NG: Drosophila melanogaster LRPPRC2 is involved in coordination of mitochondrial translation. Nucleic Acids Res 42: 13920-13938, 2014.

25. Pavlova NN and Thompson CB: The emerging hallmarks of cancer metabolism. Cell Metab 23: 27-47, 2016.

26. Zong WX, Rabinowitz JD and White E: Mitochondria and cancer. Mol Cell 61: 667-676, 2016.

27. Oran AR, Adams CM, Zhang XY, Gennaro VJ, Pfeiffer HK, Mellert HS, Seidel HE, Mascioli K, Kaplan J, Gaballa MR, et al: Multi-focal control of mitochondrial gene expression by oncogenic MYC provides potential therapeutic targets in cancer. Oncotarget 7: 72395-72414, 2016.

(i) (9) This work is licensed under a Creative Commons (c) ${ }_{\mathrm{EY}}$ No ND Attribution-NonCommercial-NoDerivatives 4.0 International (CC BY-NC-ND 4.0) License. 